\title{
Scripta
}

Revista Internacional de Literatura i Cultura Medieval i Moderna

\section{La torna de los clásicos valencianos a la llingua asturiana}

\section{The translation of the Valencian classics into the Asturian language}

\author{
Pablo SuÁrez García* \\ psuarez@coit.es
}

Academia de la Llingua Asturiana

Resume: L'artículu presenta l'estáu actual de les tornes de les obres clásiques valencianes que l'autor foi fayendo a la llingua asturiana..

Pallabres-clave: torna, Valencia, asturianu, catalán, valencianu

Abstract: This paper presents the current state of the translations of the valencian classics that the author was making into the Asturian language.

Keywords: translation, Valencia, Asturian language, Catalan language, Valencian language.

* http:/ /orcid.org/0000-0002-7085-9561. 


\section{Entamu}

Abenayá que me propunxi como xera la torna sistemática de los clásicos cimeros de la Corona d'Aragón a la llingua asturiana y, n’especial, la de los clásicos valencianos (Suárez García 2015). Ello llevóme con posterioridá a esparder l’interés inicial al casu de los clásicos castellanos y, finalmente, al de los clásicos universales.

El criteriu xeneral de toes estes tornes foi: 1) Usu d'un testu base críticu. 2) Rigor filolóxicu. 3) Caltenimientu, dientro de lo posible, del 'estilu' del testu. 4) Abondanza de notes a pie de páxina. 5) Amestadura d'entamos qu'esclarien aspeutos de la obra en cuestión o'l so autor. 6) Amestadura de bibliografia actualizao. Per otru llau, los principios que quixi siguir, en términos xenerales, quedanon reflexaos nun artículu de Lletres Asturianes (Boletín Oficial de l'Academia de la Llingua Asturiana), de mano dedicáu al Tirán el Blancu, pero que valió de guía nes restantes tornes (Suárez García 2010).

Úfrese darréu un resume de les tornes al asturianu de los clásicos valencianos que, dientro la llinia que toi acabante trazar, fixi y asoleyé yá (ap. 2); de les yá feches pero inda non asoleyaes (ap. 3); d'otres tornes yá asoleyaes nel contestu de la Corona d'Aragón (ap. 4); d'otres feches pero non asoleyaes inda, nel contestu de la Corona d'Aragón (ap. 5); y, finalmente, d'otres tornes de clásicos de la lliteratura (ap. 6). L'artículu fina con delles conclusiones (ap. 7).

\section{Tornes de clásicos valencianos asoleyaes hasta güei}

Les tornes de mio qu'hasta güei s'asoleyanon son les siguientes:

\section{a. Tirán el Blancu}

Esta torna foi Premiu d'Investigación Llingüística de l'Academia de la Llingua Asturiana nel añu 2009. Tolo que se refier a la so fechura quedó conseñao nun artículu espublizáu por Lletres Asturianes (Suárez García 2010). Foi coedición de l'ALLA y l'AVL (Martorell 2010), inda que nel fondu de la operación tevo l'IVITRA, por mor d'un conveniu de collaboración cola AVL. Les ilustraciones de les portaes fíxoles el perconocíu ilustrador asturianu Neto. La supervisión de la traducción per parte de l'AVL tevo a cargu'l profesor Albert Hauf, que calificóla de 'escelente'. La obra presentóse nes Xornaes d'Estudiu de l'ALLA 2010, con presencia del presidente y la expresidenta de l'AVL.

Inxero darréu'l fragmentu d'aniciu de la Dedicatoria del Tirán:

\footnotetext{
A honor, lloor y gloria de Nuesu Señor Dios Xesucristo y de la gloriosa sacratísima virxe María, ma de so, señora nuesa, entama la carta del presente llibru tituláu Tirán el Blancu, empobinada por Mosén Joanot Martorell, caballeru, al serenísimu Príncipe Don Fernando de Portugal. Mui escelente, virtuosu y gloriosu Príncipe, Rei espeutante:
} 


\begin{abstract}
Magar que per aciu de la sopelexada fama fora informáu de les vueses virtúes, mui mayormente agora tuvi anuncia d'aquelles, pola mor de la vuesa señoría querer comunicame y asoleyame los vuesos virtuosísimos pruyimientos sobre los fechos de los antiguos virtuosos y en fama mui gloriosos caballeros que los poetes y hestoriales aponderaron nes sos obres, perpetuando les sos alcordances y virtuosos actos. Y singularmente los mui insignes actos de caballería6 d'aquel tan afamáu caballeru que, como’l sol resplande ente los otros planetes, asina resplande esti en singularidá de caballería ente los otros caballeros del mundu, llamáu Tirán el Blancu, que pela so virtú conquistó bien de reinos y provincies dándo-yoslos a otros caballeros, nun queriendo d’ello sinón el solu honor de la caballería. Y más alantre conquistó l’imperiu griegu ensembre, arrampuñándo-yoslu a los turcos que lu soxugaran al so dominiu de los cristianos griegos.
\end{abstract}

\title{
b. Proses profanes (Joan Roís de Corella)
}

Inxero na mio torna, asoleyada pola editorial Trabe (Roís de Corella 2015), les siguientes obres de Corella:

\section{Proses mitolóxiques:}

- Razonamientu de Telamón y d'Ulises nel sitiu de Troya, delantre Agamenón, desque muertu Aquiles, sobro les sos armes

- Llantu doliosu de la reina Hécuba razonando la muerte de Príamo, la de Políxena y la d'Astianacte

- La hestoria de Lleandro y Hero

- El xardin d'amor (Llamentaciones)

- Llamentación de Biblis

- Escribe Medea a les muyeres la ingratitú y desconocencia de Xasón, pa da-yos exemplu d'honestamente vivir

- Parllamentu o collación que dempués de cenar asocedió en casa de Berenguer Mercader ente dellos homes d'estáu

- Cartes d'Aquiles y Políxena

- El xuicin de Paris

\section{Obres amoroses:}

- Traxedia de Caldesa. Razonando un casu afortunáu que con una dama-y asocedió

- Carta consolatoria a un namoráu desgracián

- Alderique epistolar ente mosén Corella y el principe de Viana

- Cartes a Yolant Durleda [atribuyida]

Obres filosófiques:

SCRIPTA, Revista internacional de literatura i cultura medieval i moderna, núm. 10 / desembre 2017 / pp. 48-66 ISSN: 2340 - 4841 doi:10.7203/SCRIPTA.10.11074 


\section{- Trunfu de les muyeres \\ - Carta qu'Honestá escribe a les muyeres}

Pa les proses mitolóxiques, arriendes de Verger (Roís de Corella 2004b) (qu’inxer unes poques proses), tuvi en cuenta principalmente los testos afitaos pola edición crítica de Martos (2001), que me permitió faer bien d'igües, y tamién los de Miquel i Planas de 1913 (Roís de Corella 1913). Tanto de la edición de Verger como de la de Martos, emplegué con profusión les notes que m’abultanon oportunes col envís d'aclariar aspeutos de la traducción. El Trunfu de les muyeres y la Traxedia de Caldesa remanecen tamién de Verger (Roís de Corella 2004b). La Carta consolatoria remanez de Martos 2005. Pal restu de los testos, la edición de partida pa la mio torna foi la clásica de Miquel i Planas de 1913 (Roís de Corella 1913). No que fai a les cuestiones téuniques de la torna, guiéme pelos mesmos principios teóricos que na mio torna del Tirán el Blancu (Martorell 2010), como yá dixi enantes (Suárez García 2010).

Previamente a esta edición, asoleyárase'l testu de la Traxedia de Caldesa, dientro de Cortijo y Martines 2013. Tamién s'asoleyara'l testu de la Hestoria de Lleandro y Hero (Cortijo y Martos 2016), nuna edición que pudi cotexar cola nueva de Martos gracies nuevamente a l'amabilidá l'IVITRA. Estos testos inxiriénonse darréu, como toi acabante dicir, na mio edición definitiva. Hebo un intentu previu d'aproximación ente l'IVITRA y l'ALLA col oxetivu d'asoleyar esta obra que, finalmente, como dixi, nun llegó a pintoriu, por mor del curtiu presupuestu de l'ALLA. De fechu, la dixebra del bloque de les Proses profanes nun volume fora una suxerencia de Vicent Martines. Finalmente, ufrióse a editala la editorial Trabe, como yá dixi.

Amueso darréu un fragmentu col entamu del Razonamientu de Telamón y d'Ulises.

Muertu'l gran fíu de Tetis, andancia espantible de los troyanos, cárcova y segura defensa de les tiendes griegues, tremontana firme de noble victoria, quien, munches vegaes del real sangre de Príamo tiñendo los campos troyanos, la granible Hécuba fixera cuasi estéril, poblando cada día los infiernos de nobles ánimes troyanes, aquel, quien Xúpiter nun osó enxendrar, temiendo la respuesta de Proteo, armáu de les fuertes armes de Vulcano, bañáu nel triste champal que por mor d'esti xuramentu los dioses non poco tarrecen. A la fin, vencíu pola estrema formosura de Políxena, el vencedor de tolos homes foi muertu, con sola una flecha aventada pol flacu brazu d'Alexandro Paris. Y, quemáu'l so cuerpu, otro nun quedó en mundu del fuerte Aquiles, sinón una tan poca ceniza, que malapenes abasta a enllenar una non mui grande vasía, y les sos armes güérfanes Ulises y Telamón con igual instancia demanden, dando por bono'l sabiu duque Agamenón que, delantre d'él, en xunto colos otros duques y reis, caún d'ellos razonara con qué drechu d'aposiar tales armes se fai dignu. Y, xirando los güeyos Telamón a les naves griegues, con soberbiosa ira dio entamu a tales pallabres:

«Oh, inmortal Xúpiter! Si, en xunto coles otres virtúes, xusticia en ti reposa, ¿comportes que delantre les nueses naves Ulises conmigo ose contender, quien, nun duldando dar llugar a les llapes troyanes, foi forciao, pa salvación de toa Grecia, que yo solu contrastara l'animosa fuerza d'Héctor, apagando'l fueu de les nueses yá quemantes fustes? Mas dexaré’l trabayu de recitar los mios actos a la fama, pues ella por min n’eterna alcordanza los recita. Diga Ulises los sos, que pa la verdá d'ellos tien mester de testigu. Yo nun inoro nin menos niego les armes d'Aquiles ser gran premiu, pero fixo poco pa demandales Ulises, quien tanto los peligros 
tarrez, que les muries nun-y paecen armes sólides. Y, agora, desarmáu, conmigo osa contender, pues con pallabres ye a algamase la fin de la nuesa contienda. Y nun ye a dir pela vida ensin gloria, que, perdida la esperanza de lo que demanda, tará allegre con que con Telamón tenga cuntes. Y, si por propies virtúes vos paez la nuesa xusticia duldosa, siendo yo nietu’l gran Xúpiter, la claridá del mio llinaxe y l'averáu deldu que teo con Aquiles, declara qu'Ulises llueu la esperanza pierda de tales armes. ¿D’ú te vien a ti, Ulises, fíu’l lladrón Sísifo, al qu'en lladronicios nun desemeyes, tener deldu col noble y real sangre d'Aquiles?»

\title{
d. Curial ya Güelfa
}

Sofitéme nel testu de la edición crítica del profesor Antoni Ferrando, de la Universidá de Valencia (Ferrando 2007), testu que tuvienon a bien apurrime l'IVITRA y el propiu Ferrando. La edición (Àvalos 2016) inxer un estudiu introductoriu de Ferrando, que torné al asturianu, y que revisó darréu'l propiu autor. Esti estudiu ye, actualizáu, el mesmu (nes estremaes llingües) que l'IVITRA punxo a toles traducciones que se fixenon nel marcu de los sos proxeutos (francés, inglés, italianu,...).

Magar la predisposición a la so coedición per parte l'IVITRA, costóme atopar editor. La edición corrió a cargu, finalmente, de la editorial Trabe n'esclusiva, magar hebo un intentu de coedición col IVITRA que nun se llogró.

\section{Úfrese darréu l'entamu del Curial ya Güelfa:}

\begin{abstract}
[1.0. PROEMIU]
¡Oh, qué grande ye’l peligru, cuántes son les solicitúes y les congoxes pa los qu'anden al aldu l'amor! Desque, magar que dellos amaos pola fortuna, dempués d'infinitos infortunios, lleguen al puertu por ellos naguáu, tantos sicasí son los que con razón se duelen por mor d'ello, que malapenes so creer qu'ente mil desventuraos s'atope ún qu'empobinara la so causa a gloriosa fin. Y si con drechu xuiciu se xulga'l casu siguiente, inda que sedrán munchos los que dirán qu'ellos quixeran qu'asina-yos asocediera colos sos amores, sicasí, sabiendo la certenidá de les penes de les qu'aquel dulzor amargoso ta dafechu enlleno, y nun habiendo certenidá de la fin si sedrá próspera o adversa, deberíen guardase muncho de metese nesti amorosu, enantes dolorosu, camín. Y poro, quiero recitavos cuánto costó a un xentil caballeru y a una noble dama l'amase ún al otru, y cómo con gran trabayu y pena, y siguíos de munchos infortunios, dempués de llargu tiempu consiguienon el gallardón de los sos trabayos.

[1.1. NEÑEZ DE CURIAL]

Hebo yá cuantayá, segun yo lleí [...], un xentil home, llamáu [...], que foi dotáu más de xacíu y de gracia de les xentes que non d'aquellos bienes qu'a los homes pal usu común la fortuna encomienda, desque solamente yera señor d'una casa baxa. Y teniendo una formosísima dama por muyer, llamada Honorada, desenguedeyaos de los trasiegos mundanales, vivíen probe y honestamente; tol tiempu, sicasí, trabayaben por algamar la gracia'l piadosu Redentor, que d'ello más que de denguna otra cosa habíen procuru continuu. Y magar que na so mocedá nun tuvieran fíos, nuesu señor Dios quixo consolalos na so vieyera, y asina, dio-yos un fíu, al que llamanon Curial, creatura según la so mui tienra edá más formosa qu’otra; y con él el pá y la ma vivíen tan contentos, asina como aquellos que muncho lu desearan, que por otra cosa del mundu más contentos nun podríen tar. Y esti neñu, pocos años dempués de la so nacencia, morriendo so pá, quedó güérfanu.
\end{abstract}




\section{e. Obra completa (Ausiàs March)}

La traducción de la Obra completa de March fíxila dende la edición crítica d'Archer (March 1997). Inda que, de mano, completara una versión filolóxica ensin escansión, mui apegada al orixinal, y que pudo paeceme, de so, interesante, abultóme dempués que podía ser más importante una versión n'endecasílabos. Esta última torna foi finalmente asoleyada pola editorial Saltadera d'Antón García l'añu 2017. Prescindióse na edición d'un notable aparatu d'anotaciones qu'acompañaba a la traducción por un motivu fundamentalmente económicu, pero tamién por mor del criteriu personal del editor, a quien nun convencen n'escesu, en xeneral, les notes a pie de páxina nes traducciones.

Ufro darréu, a mou d'exemplu, el Poema III:

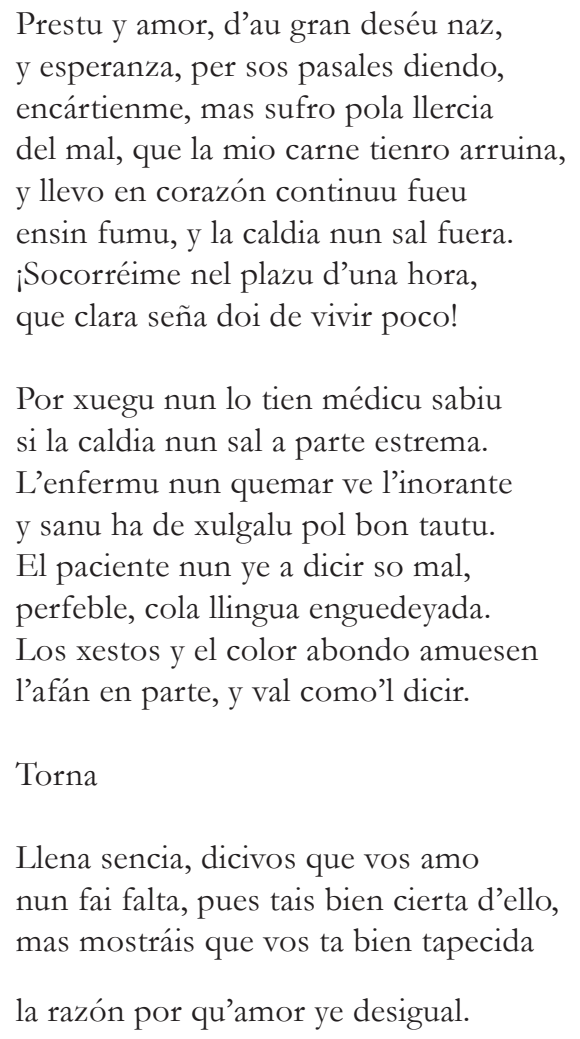

\section{Tornes de clásicos valencianos yá acabaes pero ensin asoleyar}

Les siguientes tornaes de clásicos valencianos tán peracabaes, pero téngoles anguaño nel 'caxón' de lo disponible, énte la dificultá d'atopar un editor que s’interese por elles. Son les qu’inxero darréu:

Proses non profanes (Joan Roís de Corella) 
La mio torna de les Proses non profanes de Corella nun s'asoleyó inda nin, por desgracia, atopé de momentu editor pa ella. Inclúi les siguientes composiciones íntegres:

\section{$\underline{\text { Salteriu }}$}

\section{Obres haxográfiques:}

- La hestoria de la glosiosa Santa Madalena

- La vida de la gloriosa Santa Ana

- La bestoria de Xosé, fín del gran patriarca Xacob

Otres obres relixoses:

- La visión na puerta de la Señora nuesa de gracia

- Sepultura de mosén Francí d'Aguilar

Inxer, polo tanto, la práutica totalidá de les proses relixoses espublizaes de Corella, inda qu'escluyí arréimente d'ella Lo Cartoixà (El Cartuxán), torna de Corella, como se sabe, de la Vita Christi de Ludolf de Saxonia, por mor de la escesiva estensión de la obra y pola dificultá añedida de tener que tornar el testu dende los incunables orixinales, lo qu'allargaría n'escesu'l mio trabayu.

El Salteriu remanez de la trescripción de Joan A. López i Quiles y Vicent Ribes i Palmero (Roís de Corella 1985). Del trabayu de López i Vicent tamién tomemos material abondo de les sos notes. La visión na puerta de la Señora nuesa de Gracia tomémosla de Wittlin (1995).

Pal restu de los testos, la edición de partida pa la mio torna foi la clásica de Miquel i Planas de 1913 (Roís de Corella 1913).

\section{Vien darréu la Forma de la sepultura, de Sepultura de mosén Francí d'Aguilar.}

Empobiné la vista, de los otros sentimientos talaya, a aquella parte, d'au l'oyíu xulgaba que tan seles voces resonando veníen. Y fonon los mios güeyos illuminaos de claridá tan perfeuta, que yo estimé qu'Apolo, dexáu l'acostumáu círculu, recordaba del tiempu del noble duque Xosué, la estación almirable; y yera la escelsa claridá asina de perfeición alta, que la débil humana vista fortificando confortaba, apurriéndome puxu pa más cerca averame a la puerta de la santa capiella. Nun foi grande'l númberu de los atentaos pasos, cuando a la illuminada vista se presentó una magnífica sepultura, asina d'altamente fabricada, que nun fora posible qu'humanes manes acabala pudieran; y taba sobro l'alabastru, esculpíu, del vivu en figura humana, d'un caballeru la imaxe, en forma non mui alta, d'elegante proporción, la cara venusta, d'aquella edá que, nel postrer día, los bonaventuraos eleutos cobrarán glorificaos los propios cuerpos. D’armes d'España tenía la persona cubierta; la espada na mano, que la mayor parte d'ella taba, de frescu corriente sangre, roblada d'ardiente carmesín, que les brases enceses en color vencía. Anubriendo la esculpida sepultura, un ricu pabellón n’alto s'elevaba, semáu, en perelevada orfebrería, de los árboles de la mirra; y, nes fueyes, esmaltaes lletres ensin dificultá se lleíen: «Milicia est uita homims super terram». Y yera del bordáu pabellón la flocadura, d’oru estirao, en lletres figurada: «Preciosa 
Pablo Suárez García. La torna de los clásicos valencianos a la llingua asturiana

in conspectu Domini mors santorum eius». Que sobro esta sele harmonía, un águila les ales batía, qu'esnalando al rodiu la clara sepultura, sobro’l pomu del ricu pabellón se posaba.

\section{b. Poesía completa (Joan Roís de Corella)}

Como nel casu anterior, nun atopé inda editor pa la Poesía completa de Corella. La torna fíxila en versos endecasílabos, siguiendo’l modelu qu'emplegué nel casu de March. Inxer les siguientes composiciones:

\section{$\underline{\text { Relixosa: }}$}

- Vida de la sacratísima Virxe María, Ma de Dios, Señora Nuesa

- Respuesta con versos llibres en lloor de la Virxe María, tirando a la xoya

- Oración a la sacratísima Virxe María, teniendo'l so fiu Dios Xesús na falda amiyáu de la cruz

\section{Amorosa:}

- A Caldesa

- La Sepultura (Describe la sepultura de la so namorada)

- Corazón cruel (A la so namorada)

- La balada de la pega y la mierla

- Llantu d'amor

- Desengañu (Los qu'amáis)

- Somisión amorosa (Nun foi tan grande de los xudíos el temor)

- Copla esparza

- La muerte por amor (Copla esparza)

- Recuesta d'amor [atribuyida]

- Debate con Caldesa [atribuyida]

D'alderique:

- Imperfeición bumana

- Flores del saber

¿¿Ye a matar piedá?

SCRIPTA, Revista internacional de literatura i cultura medieval i moderna, núm. 10 / desembre 2017 / pp. 48-66 ISSN: 2340 - 4841 doi:10.7203/SCRIPTA.10.11074 
El puntu de partida pa les poesíes de Corella fonon les ediciones de Verger (Roís de Corella 2004 y 2004b), inda que tamién tuvi en cuenta dellos apeutos inxeríos en Martines Peres 1999. El poema que titulé Epitafiu remanez de Martos 2008. Per otru llau, no que fai al títulu de los poemes, caltuvi en xeneral el que-ys dio la tradición.

En tou casu, los poemes van acompañaos de la so versión orixinal, lo que permitiría al llector faese una idea del mayor o menor aciertu na nuesa xera de traducción. Tamién s'inclúin un númberu notable de notes.

Inxero, darréu, el poema $A$ Caldesa:

Si’l fierru caldiu esfrez la mano casta, caldiáislu vós, magar que tea fríu; si dafechu nel mundu'l fueu se pierde, puen coyelo de vós, que dais aguantu; si, quemando, la tierra s'afarare, nun morreréis, que sois vós sacavera, nin perderéis de faca d'Eire l'usu por que dexéis la vuesa xentil casta.

Casta ha ser, abondosa, la de vueso, ensin xamás ser güérfana de pas: nu'ha abastavos el mundu pa compadres, si algo parís; según la vuesa quexa, dulda tó qu'enxamás forais donciella; dote ensin arres pide la llei vuesa; tol mundu tien del vuesu cuerpu muestra: el fiel, l'infiel y aquel de vieya Llei.

La ganzabera verde foi nes llapes, y, ensin fueu, vós tenéis calor que quema; llume han coyer, si vienen col envís d'igual que na ferrial piedra bater; «baratera muyer» por vós se dixo, que fueu lleváis embaxo de les faldes, y tol mundu, pues nun trancáis con piesllos, pue coyelo igual que na foguera.

Calda amburiante más que'l fueu na esfera, pa dicir «non» zarravos fe-y los dientes, que dicise nun pue qu'un requiriente nunca oyere enxamás de vós: «Espera». 


\section{c. Poesía completa (Jordi de Sant Jordi)}

Los testos orixinales emplegaos de mano pa la mio traducción fonon los inxertos nel Repertorio informatizzato dell'Antica Letteratura Catalana de la Universidá de Nápoles (RIALC: http://www.rialc. unina.it/inc-santjordi.htm). Posteriormente tuviénonse en cuenta mui detenidamente los testos inxertos por Fratta na so edición crítica (Sant Jordi 2005). D’esti últimu autor emplegóse también material adicional abondo pa les nueses notes.

Inxero a mou d'exemplu'l siguiente poema de Jordi de Sant Jordi:

\section{ERMU D'AMIGOS, BIENES YA SEÑOR}

Ermu d'amigos, bienes ya señor, n’estrañu sitiu y n'estraña comarca, lloñe de tou bien, fartu anoxu y murnia, mio voluntá y pensamientu cativos, sometíu m'atopo a un mal poder, quien por min s'esmoleza nun alcuentro, y toi guardáu, peslláu, ferráu y presu, y a mio triste ventura-y lo agradezo.

Vi tiempos nos que nada me prestaba, agora toi contentu atristayáu, y los grillos llixeros más m'abulten que nel pasáu la guapa bordadura. Fortuna vi amosar el so querer so min, queriendo a tal puntu treme; nun m'esmuelga, pues fixi'l mio deber colos bonos que tán na mio compaña.

Que consuélome siendo prisioneru pol mio señor, sirviéndo-y cuanto pudi, d'armes sobráu y por mayor poder, y non por falta de caballería.

Y consuélome al nun ser a algamar tener nada ensin pasar trabayos, mas d'otru llau morrer cuido de murria viendo'l mundu folgase pol contrariu.

Nada ye acostinar con estos males al llau del que'l corazu me trespasa y xebrame me fai de la esperanza: nun ver nada qu'un poco nos avance en procurar el nuesu llibramientu, y más viendo lo que nos pide Sforça, qu'a les nueses razones nun atiende, 
y mio virtú esmorez y la mio fuercia.

Poro, nin sé nin veo un res anguaño que sía a favoreceme niun migayu, nun siendo solo Dios, en quien m'encontio y esfoto, y mio corazu tien consuelu; y d'otru llau el bon rei lliberal, qu'acorrióme por so gran xentileza, quien nos punxo dafechu nesti mal, y ha sacame, pues toi baxo'l so mandu.

Torna

Rei virtuosu, mio señor natural, nada nun vos pidimos nós agora sinón que recordéis que’l sangre real nunca nun engañó al que foi de so.

\section{d. Dellos cuentos (Enric Valor)}

De momentu, completé la torna de los cuentos d'Enric Valor qu'inxero darréu: El xugador de Petrer, Abella, El neñu que nació de pies, El Castiellu de VasDiry NunVasVolver, Hestoria d'un mediu pollu. El testu base foi'l de la editorial Tàndem (Valor 1995).

Incluyo, darréu, el final del cuentu El xugador de Petrer.

[...] Y neses, llegó l'añu oncenu, esto ye, l'añu que se presentaría Cabraliegu'l Finu a llevase la so alma.

Aquel añu la collecha d'arbeyones fora magnífica. Y el demoniu, allá n’agostu, una nueche dexó la sierra de Castalla y baxó al valle de Petrer.

Paró debaxo un algarrobu ramudu. Les arbeyones taben yá madurecíes y namái tocales, daben en suelu.

Cabraliegu díxose mui floxino:

-Dientro quince díes nun va quedar dala enriba y entós llevaré l'alma d'aquel calavera de don Pere. Meyor... Daré-y un mes entá. Asina de xuru que nun habrá arbeyona que nos estorbe’l tratu.

A lo cabero d'ochobre, don Pere, acabante cenar, en tranquila compañía de tola familia, falucando de sobremesa, vio apaecer a la puerta'l comedor la elegante figura de Cabraliegu'l Finu. ¡El corazón dio-y un valtu!

Por suerte nun lu vía naide más qu'él, polo que nin la so muyer nin los fíos, naide nun fixo nengún esparabán. Viéndolu tan esmolecíu polos otros, el demoniu echóse a rir.

—Nun me ve naide más que vós, heriede don Pere. Y naide nun me siente tampoco. Si vos llevantáis y dicís que vais a casa don Antoni, la vuesa muyer nun s'estrañará un res.

Don Pere llevantóse.

—Agora m'alcuerdo — dixo pernatural—; esta nueche teo prometida una visita al mio amigu del Pla de Dalt.

— ¡Nun tardes en volver, mio esposu! — dixo perafeutuosa Maria.

Don Pere besó ún por ún fíos y fía. 
—Besái a la muyer, si vos presta — dixo irónicu dafechu Cabraliegu—, y asina tenéislos a toos despidíos. Ye doloroso, pero nun vais volver velos más nunca.

Don Pere nun s'inmutó gota y salió de la casa con capa y sombreru y siguíu del demoniu.

Peles cais nun pasaba naide.

—Daime yá l'alma —dixo'l demoniu.

—Esperái, Cabraliegu... Los tratos son tratos —opúnxose tou gayasperu y serenu don Pere.

—¿Qué tratos? ¡Yá pasa munchu tiempu del términu prometíu!

—Díi despacio, amigu! — prosiguió percortés l’heriede—. Vosotros tenéis fama de ser serios y formales.

—Yá, yá —dixo impaciente Cabraliegu—. Pero yo tengo priesa.

— ¿Y les arbeyones? Tenemos de comprobar los de les arbeyones.

—Yá nun queda dala — riól demoniu—. Baxé tres o cuatro vegaes de la sierra pa ver cómo les coyíen los llabradores.

Afilvanaron cai abaxo y salieron al camín d'Elda y caballeru y diañu pararon debaxo'l primer algarrobu que-yos aprució delantre.

—Fai bona lluna — dixo'l demoniu—. Vei si veis denguna arbeyona, nin enriba nin embaxo.

- Según a qué vos refiráis, demoniu Cabraliegu. Vós nun ye xusto que llaméis solamente arbeyones a les que vistis meter nel sacu mientres la coyida. Daivos cuenta; averáivos equí. Y l'heriede, con cara afable, amosó al demoniu les arbeyones tienres qu'había garraes per toles cañes, dende les grueses hasta les fines.

—¿Qué ye esto? — saltó’l diañu ablucáu.

—Nun se cumplió’l términu afitáu, Cabraliegu — sentenció en voz alto don Pere-, porque l'algarrobu ye un árbol que nun para enxamás: ¡cuando nun les tien madures tienles verdes!

— ¡Brrr! —entamó bramar Cabraliegu—, xugador, más que xugador, ¡fixístisme trampes en xuegu'l contratu!

Y ye qu'aquel demoniu vivía nes tierres altes, onde nun hai algarrobos, y yeren pa él cuasi per completo desconocíos, a lo menos nel detalle de la collecha.

- Si nun vos presta'l tratu, culpáilo a la vuesa inorancia — dixo don Pere, que, tiesu como un fusu, nun sabía quá facer: si marchar d'ellí como si fuxera o quedar y ver en qué paraba la cosa. Entós viéno-y a la imaxinación encomendase al patrón del pueblu, a san Bonifaciu gloriosu.

- San Bonifaciu, pá de nueso... —entamó en voz alto.

Y Cabraliegu quedó quietu y tou temblosu.

— ¡Tenéis razón, don Pere! ¡Perdí! ¡Marcho! — vocingló.

Abrióse un furacu metanes el suelu y Cabraliegu'l Finu, con una gran llaparada, desapaecío pa siempre.

Don Pere volvió adulces pa so casa. Reinaba ellí un gran silenciu. Toos dormíen un sueñu sele y esfotáu.

\section{Otres tornes yá asoleyaes de la Corona d'Aragón}

\section{a. El suañu (Bernat Metge)}

El testu base de la mio torna foi la edición crítica de Stefano Maria Cingolani de la editorial Barcino (Metge 2006). Caltuvi la división en seiciones y parágrafos nel interior de los llibros que propón Cingolani. 
Tamién tuvi en cuenta, en cuestiones de detalle, la edición de Lola Badia asoleyada por Quaderns Crema en 1999 en Barcelona (Metge 1999), que se sofitaba na de Martí de Riquer del añu 1959 d'acordies con tolos manuscritos conocíos (Riquer 1959).

La obra asoleyóla la editorial Trabe al empar que les Proses profanes de Corella (Metge 2015).

Ufro darréu l'entamu'l parllamentu d'Orfeo, nel llibru III:

(i,3) Apolo foi mio pá, y Calíope mio ma, y nací en reinu de Tracia. La mayor parte’l tiempu de la mio vida gastélo en retórica y música. Muyer tuvi mui curiosa, llamada Eurídiz, la que yera pa min más querida que la vida. Pa la so desventura, diendo folgar cerca la vera un ríu, foi de llibidinosu amor requida por Aristeo, pastor; y cuando ella, fuxendo d'aquel per un prau foi mordida y envelenada en calcañu por una culiebra equí escondida, nel intre morrió y amiyó al infiernu. (ii,4) Conocida por min la so dolorosa muerte, amiyé a les puertes d'aquel, y tocando la llira, que me diera Mercurio, fui tan graciosu a Cerbero, porteru l'infiernu, que les diches puertes fóronme aína abiertes. Dempués qu'entré dientro, constituyíu’l consistoriu de los presidentes infernales, dixi: «Si a la vuesa gran potencia nun espongo na manera que debo aquello polo que me convieno venir equí, suplico que me sía perdonao, desque afuera to del mio xacíu pol repentín ya inopináu infortuniu que sobre la mio dolorosa cabeza cayó. Nun vini equí pa mirar les tiniebles infernales, a les que necesariamente toa cosa mortal ha amiyar, nin a encadenar el pescuezu de Cerbero, asina como dellos fixenon; la sola causa de la mio venida ye la mio muyer, a quien, tando na flor de la so mocedá, una culiebra mató col so velenu. Entamé, mas nun fui quien, de sufrilo pacientemente: l'amor d'aquella vencióme. Si la fama antigua ye verdadera, toos tuviestis namoraos asina como yo; préstevos, darréu, que la dicha muyer mía queráis restituyime a min. Toles coses vos son debíes y, sero o ceo, equí debemos xeneralmente venir: tol mundu tien de perecer. Esta ye la nuesa cabera casa; vosotros aposiáis los perpetuos reinos del humanu llinaxe. Cuando la dicha muyer mía aporte a la so vieyera, tamién ha ser vuesa: solamente vos la demando pal mio usu. Nun me refuguéis lo que vos pido, desque, si lo faéis, sabéi que nun volveré d'equí, y entós folgaréisvos de la muerte de dambos».

\section{Tornes acabaes, pero non asoleyaes inda, de la Corona d'Aragón}

\section{a. Xacob Xalabín (anónimu)}

Esta torna, xunto coles de la Hestoria de la fía l'emperador Constantín y la Hestoria de la fíal rei d'Hungría (vei más alantre), foi Accésit del Concursu d’Investigación Llingüística de l'ALLA 2013. El testu base foi la edición clásica de Miquel i Planas de 1910 (Miquel i Planas 1910). Tuviénonse mui en cuenta tamién tanto la edición de Lola Badia (Badia 1986), que se sofitaba na clásica y crítica de Pacheco (Pacheco 1964), como la de Cingolani (Cingolani 2012), n'especial no que fai a la formación de párrafos y puntuación, inda que tamién fonon importantes no que fai a otros aspeutos de detalle.

La traducción foi, en tolos casos, enforma testual (y reflexa, polo tanto, de mou tresparente, rasgos d'estilu del orixinal, que quixemos caltener arréimente), inda que cola llende siempre necesaria de nun forciar n'escesu les construcciones na nuesa llingua, y que'l resultáu, en definitiva, fora correutu dende un puntu de vista gramatical. 
Emplegánonse tamién dellos estudios y monografíes adicionales de los que se fixo usu dacuando nes notes a pie de páxina, o tamién nel mesmu procesu de traducción, y que, en xeneral, quedaron reflexaos nun apartáu bibliográficu.

Preséntase darréu un fragmentu del Xacob Xalabin, de la parte que correspuende a Cómo se fixo la batalla de los Turcos y de los Cristianos y cómo morrió l'Amorat.

Y Llázaro, que taba mui deseosu de que la so xente firiera a la escontra lo del Amorat, p'hacia la hora de viéspores, el caballeru Tudescu entamó atacar a caballu con tola so xente y firir en tolos camellos que primero taben, y los caballos nun s'echanon atrás de nenguna manera, enantes firíen fuertemente de mou que rompienon los camellos y fixenon sitiu, estos tol tiempu firiendo d'espueles los caballos, de mou qu'entranon per metanes la güeste del Amorat tanto que fonon fasta les tiendes de los alachás, esto ye d'aquellos que vendíen la vianda. $\mathrm{Y}$ cuando estos fonon fasta les tiendes, xiranon los sos caballos y pensanon tornar contra aquella parte d'au vinieran y tola güeste de los Turcos fixenon sitiu nesta brañada de xente que vienon qu'entraran asina. Y asina, cuando la otra xente de Llázaro debiera entrar pa socorrer al llombu, pensanon, cuando vienon qu'aquellos tornaben, que fuxeran, y nun osanon entrar. Y naquel momentu, aquel caballeru Húngaru que xurara combatese col Almorat cuerpu a cuerpu, nun tevo curiáu de la so xente, enantes, avanzáu dafechu de los otros, afaló'l so caballu p'hacia aquella parte au pensaba que 1'Amorat s'atopaba, que taba con um pendonucu con una gran brañada de xente, y esti fixo la vía d'aquel cola espada o llanza engocetada. Y 1'Amorat, que vio venir esti caballeru asina solu cola so espada engocetada, tévolo por escarniu, y dixo a la xente qu'al rodiu taba que tol mundu-y fixera sitiu pa ver el caballeru qué fadría, y el caballeru fixo la vía del Amorat y 1’Amorat, que vio que la so vía faía, tiró-y una flecha y dempués otra y el caballeru nun-y faía dengún casu, y fixo la so vía cola llanza engocetada, y dio-y tal golpe col poder del caballu que l'adarga y unes coraces que l'Amorat tenía, too-y lo pasó, y metió-y el fierru la llanza pel costáu unos bonos cuatro deos y valtólu mui malamente mancáu en suelu como muertu, pero inda nun morriera del too.

Y la xente qu'equí taba, que vienon el so señor que xacía muertu, d'esmenu, hebo equí gran fragor y, nun momento, aflechanon el caballeru, que por esta razón per tola güeste hebo gran xaréu, y les güestes toes que se mecienon.

Y, de mou asemeyáu, a los primeros golpes que dienon, Llázaro morrió, quien se meciera nuna parte de la batalla contra Enabechsu Bey. Y cuando'l xenru de Llázaro supo que so suegru taba muertu, nun tevo procuru de firir nin de proceder na batalla, enantes mandó que nengún home nun se tascara y, darréu, ocupóse de tornar pa la so tierra pa ser señor.

\section{b. Llibru del Orde de Caballería (Ramon Llull)}

Esta torna fíxose principalmente dende la edición dixital del Institutu Joan Lluís Vives, arriendes de la versión orixinal reproducida por Cortijo na so edición (Llull 2015), inda que se tuvienon en cuenta otres versiones. Ufro darréu un fragmentu de la estaya cola qu'entama la obra:

1. Nuna tierra asocedió qu'un sabiu caballeru, que llongamente caltuviera la orde de caballería na nobleza y puxu del so altu corazón (y sabencia y ventura caltuviéranlu nel honor de caballería en guerres y en torneos, n'asaltos y en batalles), escoyó vida ermitaña desque vio que los sos díes yeren curtios y la naturaleza esfallecía-y por mor de la vieyera a la d'usar les armes.

Entóncenes abandonó les sos heredaes y dió-yosles n’herencia a los sos fíos; y nuna viesca grande, abondosa d'agües y d'árboles frutales, fixo la so habitación, y fuxó del mundu, col 
envís de que la ruindá del so cuerpu, na que cayera por mor de la vieyera, nun lu deshonrara naquelles coses nes que la sabencia y la ventura per llargu tiempu lu caltuvieran honráu. Y, poro, el caballeru pensó na muerte, recordando'l pasamentu d'esti sieglu al otru, y entendió la sentencia perdurable a la qu'había llegar.

2. Nun prau prestosu había un árbol pergrande, encorrompináu dafechu de fruta, onde’l caballeru vivía naquel monte. Embaxo aquel árbol había una fontana mui guapa y clara, y por mor d'ella yera abondosu'l prau y los árboles que taben al rodiu.

Y el caballeru tenía por costume tolos díes venir a aquel llugar a adorar y contemplar y rogar a Dios, al que daba gracies y mercedes pol gran honor que-y fixera tol tiempu de la so vida nesti mundu.

\section{c. Dellos cuentos medievales}

Un par de cuentos anónimos medievales: Hestoria de la fía l'emperador Constantín y Hestoria de la fíal rei d'Hungría fonon, xunto col Xacob Xalabin, Accésit del Concursu d'Investigación Llingüística de l'ALLA 2013, como yá dixera. No que fai al primer cuentu, el testu base foi la edición clásica de Miquel i Planas de 1910 (Miquel i Planas 1910). No que cinca a la Hestoria de la fíal rei d'Hungría, fíxose a partir de la inxerida por Miquel i Planas n’otru volume tamién de 1910 (Miquel i Planas 1910b).

Ufro darréu un fragmentu de la Hestoria de la fía'l rei d'Hungría, aquel nel que corta les sos manes:

Con esto, el rei partióse de la donciella, y ella quedó con gran pensamientu, y rogó a Nuesu Señor qu'él la guardara pa qu'aquel pecáu nun tuviera acabamientu. Y cuando vieno l'atapecer, que toos s'achucanon, ella fixo pesllar la puerta la so cámara, y apelló una dueña126 que taba con ella de camarera, y dos doncielles. Y díxo-ys:

- ¿Vosotres xuráisme que fadréis lo que yo vos diga?

- Sí, mio señora —y xuránon-ylo.

Dixo la fía'l rei:

- Mio pá quier garrame por muyer por malos conseyeros que tien, y diz que ta namoráu de min especialmente pola formosura les manes, y yo quiero más perder les manes

que non perder la mio virxinidá nin consentir a tan grande pecáu. Polo que dígovos y mándovos, pol sagramentu127 que me fixestis, que me cortéis les manes.

Cuando elles oyenon esto, entamanon llorar y dixenon que por nada del mundu nun lo fixeran, qu'enantes se dexaran matar.

—Sabéi — dixo la donciella— que, si nun lo faéis, yo llamaré, y fadrévos quemar por mio pá, y arrastrar.

Y desque elles oyenon esto, sintienon llercia de ser arrastraes y quemaes. Atánon-y les manes la una sobre la otra, y mangóles ella mesma sobre'l cepu, y una donciella caltuvo'l cuchiellu sobre les manes y, la otra, con un mayu, firió sobre'l cuchiellu. Y, asina, con gran dolor y con grande pena, cortánon-y les manes. Y garranon llueu un fierru ingrientu y, asina como ella lo ordenara, cauterizánon-y los tucos.

Fecho esto, fixo meter les manes nun formosu tayaderu d'arxentu y fixo cubriles con una guapa toballa de seda, y ella fixo metese en llechu y abrigar bien. Tantu foi'l dolor qu'ella sufría que nengún home nun pudiera pensalo, mas ella sola lo sabía, la verdá qu’elles sentíen. 


\section{Otres tornes de clásicos}

Les siguientes tornes de nueso, yá asoleyaes, vienen completar el carapiellu d'obres clásiques n’asturianu feches hasta güei por min:

L’inxeniosu fidalgu Don Quixote de la Mancha, asoleyáu pola editorial Laria en 2015. Cola nuesa torna ufrimos una versión íntegra — la primera n'asturianu — del Quixote de Cervantes. Inda que, de mano, partiemos del testu inxertu na Biblioteca Virtual Miguel de Cervantes sofitáu na edición de Madrid, Ediciones de la Lectura 1911-1913, esta versión de partida foi cotexada y detenidamente iguada puntu per puntu coles ediciones crítiques de Rico de 1998 y 2011, de manera que podemos dicir que'l nuesu testu base definitivu correspuéndese más bien cola edición crítica de Rico. Por supuesto, consultemos, arriendes, yá en determinaes cuestiones puntuales, otres ediciones estremaes del Quixote, como, por exemplu, la de Castalia de 1998.

Llazarín de Tormes, asoleyada pola editorial Laria en 2017. El testu base d'esta edición foi'l de la edición de Burgos (1554), al que s'amestanon interpolaciones remanecíes del de la edición d'Alcalá (1554), y que se marcanon na nuesa versión per aciu de llaves $(\{\ldots\})$ y de cursives. Consultánonse, arriendes, delles ediciones modernes, ente elles, fundamentalmente, la de Francisco Rico (asoleyada por Cátedra en 1987), inda que se remananon otres.

Les siguientes tornes, a les que yá punxi'l ramu, van espublizase, finalmente, a lo llargo l'añu que vien (2018), darréu que yá esisten alcuerdos editoriales al respeuto:

La Celestina va asoleyala la editorial Laria dientro la coleición "Clásicos n'asturianu", na que s'inxirienon tamién les mios traducciones del Quixote y el Llazarín de Tormes. Na nuesa torna empleguemos como testu base La Celestina espublizada col procuru de Julio Cejador y Frauca en 1913 en Madrid por Ediciones de la Lectura, dientro la coleición Clásicos castellanos. El testu de Julio Cejador taba sofitáu principalmente na edición de la Librería de Eugenio Krapf, Vigo del añu 1900, que siguió a la so vez la de Valencia de 1514 (y que yera reproducción de la de Salamanca de 1500). Julio Cejador tevo mui en cuenta tamién na so edición la de Burgos de 1499, espublizada por Foulché-Delbosc nel 1902. El testu base foi cotexáu darréu col de la edición de la RAE de 2011, inxiriendo nél les modificaciones que nos abultanon afayadices, y garrando d'aquella edición un bon númberu de notes a pie de páxina, que camentemos que podríen resultar del interés del llector asturianu.

No que fai al Decamerón, va asoleyalu la editorial Trabe. La torna al asturianu fíxose dende la versión toscana orixinal, emplegando como testu base la magnífica edición del Decameron de Giovani Boccaccio asoleyáu pola editorial Mondadori, col procuru de Vittore Branca, en Milán, en 1985. Col oxetu d'aclariar estremaos aspeutos de la obra, emplegánonse, adicionalmente, otres munches ediciones anotaes que se fixenon constar puntualmente nes notes a pie de páxina. 


\section{Conclusiones}

El númberu de les mios tornes asoleyaes al asturianu d'obres cimeres valencianes en particular, y pertenecientes a la Corona d'Aragón en xeneral, va medrando adulces. Tamién el de les mios tornes de clásicos castellanos y propios d'otres lliteratures. Ye importante'l fechu del espardimientu n'Asturies d'autores munches vegaes desconocíos que, de dalgún mou, tán calistrando y fayéndose sitiu nel universu de la lliteratura n'asturianu. L'impautu de la entrada d'autores y obres desconocíos ye, como se sabe, enforma mayor que'l de los que se conocen bien per llectura direuta o bien per aciu del enseñu. De fechu, suel ser esti criteriu perimportante d'escoyeta d'obres a asoleyar no que fai a bien d'editoriales.

Per otru llau, ye evidente que los enzancos económicos pelos qu'hai d'arreblagar son importantes pa continuar cola xera, lo que lleva dacuando a un tiempu d'espera relativamente llargu hasta l'asoleyamientu d'una torna desque acabada. De toles maneres, paez qu'hasta agora, adulces, fonon solventándose estes estorbises, gracies a instituciones como l'ALLA, l'AVL, l'IVITRA, y a editoriales privaes como Trabe, Saltadera o Laria.

\section{Bibliografia y sigles}

ALLA = Academia de la Llingua Asturiana.

ALTLLEV $=\underline{\text { http://pablosuarezgarcia.blogspot.com.es/. }}$. Blogue lliterariu del autor d'esti artículu.

(Anónimu). Llazarín de Tormes. Torna al asturianu de Pablo Suárez García. Uviéu, Laria, 2016.

ÀVALOS, Íñigo d' (2016). Curial ya Güelfa. Torna al asturianu de Pablo Suárez García. Uviéu, Trabe, 2016.

AVL $=$ Acadèmia Valenciana de la Llengua.

Badia, Lola (ed.) Història de Jacob Xalabin. 3er edición. Barcelona, Ediciones 62, 1986.

Cervantes Saavedra, Miguel de (2015) L'inxeniosu fidalgu Don Quixote de la Mancha. Torna íntegra al asturianu de Pablo Suárez García. Uviéu, Laria, 2015.

Cingolani, Stefano Maria (ed.) Història de Jacob Xalabin. Barcelona, Ediciones 62, 2012.

Cortijo Ocaña, Antonio \& Martines, Vicent (eds.) (2013) Multilingual Joan Roís de Corella. The Relevance of a Fifteenth-Century Classic of the Crown of Aragon / Joan Rois de Corella Multilingüe. La importància d'un clàssic de la Corona d'A ragó del segle XV. California, Santa Bárbara/Publications of eHumanista, 2013. 
Cortijo Ocaña, Antonio \& Martos, Josep Lluis (eds.) (2016) The Story of Leander and Hero, by Johan Rois de Corella / A multilingual edition of a classic from the Crown of Aragon. Amsterdam, John Benjamins, 2016.

Ferrando Francés, Antoni (ed.) Curial e Güelfa. Introducción y edición filolóxica. Toulouse, Anacharsis Editions, 2007.

Llull, Ramon (2015) The Book of the Order of Chivalry / Llibre de l'Ordre de Cavalleria / Libro de la Orden de Caballería. Introduction and translation into English and Spanish by Antonio Cortijo Ocaña. IVITRA Research in LingUistics and Literature. Amsterdam: John Benjamins.

Llibre de l'Orde de Cavalleria. Edició digital de l'Institut Joan Lluís Vives.

March, Ausiàs(1997) Obra completa. Edición de Robert Archer. Barcelona, Editorial Barcanova, 1997.

(2017) Obres completes. Torna al asturianu de Pablo Suárez García. Uviéu, Saltadera, 2017.

Martines Peres, Vicent (1999) «Sin adobo se podrán bien servir. Traducción y filología: traducción al español de la lírica de Joan Rois de Corella». Revista de Filología Románica, 16, páxs. 213-264, 1999.

Martorell, Joanot (2010) Tirán el Blancu. Torna íntegra al asturianu de Pablo Suárez García. Uviéu, ALLA, 2010.

Martos, Josep Lluis (ed.) (2001) Les proses mitològiques de Joan Roís de Corella. Edició crítica. Barcelona, Publicacions de l'Abadia de Montserrat, 2001. 2008.

(2008) «La literatura perduda de Joan Roís de Corella: les fonts». Caplletra, 45, páxs. 93-112,

Metge, Bernat (1999) Lo somni. Edición de Lola Badia. Barcelona, Quaderns Crema, 1999.

(2006) Lo somni. Edición de Stefano Maria Cingolani. Barcelona, Barcino, 2006.

(2015) El suañu. Torna al asturianu de Pablo Suárez García. Uviéu, Trabe, 2015.

Miquel i Planas, Ramon (ed.) (1910) La Història de Jacob Xalabin seguida de la de La filla de l'emperador Contastí. En: Històries d'altre temps. Textes catalans antics, VII. Barcelona, 1910.

(ed.) (1910b) La Història de Valter e Griselda arromançada per Bernat Metge seguida de les de La filla del rey d'Hungria, Frondino e Brisona y Paris e Viana. En: Histories d'altre temps. Textes catalans antics, I. Barcelona, 1910b.

Pacheco, Arseni (ed.) La Història de Jacob Xalabin. Barcelona, Barcino, 1964.

Riquer, Martí de (1959) Obras de Bernat Metge. Barcelona, Universitat de Barcelona, 1959.

Roís de Corella, Joan (1913) Obres de J. Roiç de Corella, publicades amb una introducció per R. Miquel y Planas segons els manuscrits y primeres edicions. Barcelona, Biblioteca Catalana, 1913.

(1980) Tragèdia de Caldesa i altres proses. Barcelona, Edicions 62, 1980.

SCRIPTA, Revista internacional de literatura i cultura medieval i moderna, núm. 10 / desembre 2017 / pp. 48-66 ISSN: 2340 - 4841 doi:10.7203/SCRIPTA.10.11074 
Pablo Suárez García. La torna de los clásicos valencianos a la llingua asturiana

(1985) Psalteri. Introducció, transcripció i actualització a cura de Joan A. López i Quiles i Vicent Ribes i Palmero. Revista Saó, Publicacions de l'Abadia de Montserrat. Valencia, Conselleria de Cultura, Educació i Ciència de la Generalitat Valenciana, 1985. (1985b) Poesia i prosa. Barcelona, Edicions 62/Orbis, 1985b.

(1998) Leandre $i$ Hero. Història de Josep. Versión y actualización de Josep Lozano y Magda Añón. Alzira, Bromera, 1998. 2001.

(2001) Prosa profana. Torna de Vicent Martines. Clásicos Medievales, 21. Madrid, Gredos,

(2004) Poesías. Edición y torna [al castellán] d’Eduard J. Verger. Colección Calabria / Poesía. Valencia, Editorial Denes, 2004.

(2004b) Poesies $i$ algunes proses. Entamu, edición y notes d'Eduard J. Verger. Espublizada na rede so llicencia Creative Commons, 2004b.

(2015) Proses profanes. Torna al asturianu de Pablo Suárez García. Uviéu, Trabe, 2015.

Sant Jordi, Jordi de (2005): Poesies. Edición crítica d'Aniello Fratta. Col. B, númb. 26, Barcelona, Barcino.

Suárez García, Pablo (2015) «La torna de los clásicos de la Corona d’Aragón a la llingua asturiana». Mirabilia/MedTrans, númb. 2015/2.

(2010) «La torna del Tirant lo Blanch al asturianu». En: Homenaxe al profesor García Arias. Lletres Asturianes, Anexu I, páxs. 977-1010. Uviéu, ALLA, 2010.

Valor, Enric (1995) Rondalles valencianes d'Enric Valor. Adaptació de Rosa Serrano. Valencia, Tàndem, 1992-1995.

Wittlin Curt (1995) «Un text inèdit de Joan Roís de Corella: La visió a la porta de la Senyora nostra de Gràcia». En: A sol post, núm. 5, Estudis de llengua i lliteratura 3, páxs. 257-268, Coleición Universitas. Alcoi, Marfil, 1995. 